\title{
Fisioterapia no Paciente sob Ventilação Mecânica
}

\author{
Physiotherapy on the Mechanically Ventilated Patients
}

\author{
George Jerre', Marcelo A. Beraldo², Thelso de Jesus Silva ${ }^{3}$, Ada Gastaldi', Claudia Kondo", \\ Fábia Leme ${ }^{6}$, Fernando Guimarães ${ }^{7}$, Germano Forti Junior ${ }^{8}$, Jeanette J. J. Lucato ${ }^{9}$, \\ Joaquim M. Veja ${ }^{10}$, Alexandre Luque ${ }^{11}$, Mauro R. Tucci'12, Valdelis N. Okamoto ${ }^{13}$
}

\section{RESUMO}

JUSTIFICATIVA E OBJETIVOS: Em 2000, foi publicado o II Consenso Brasileiro de Ventilação Mecânica. Desde então, o conhecimento na área da ventilação mecânica avançou rapidamente, com a publicação de inúmeros estudos clínicos que acrescentaram importantes informações para o manuseio de pacientes críticos em ventilação artificial. Além disso, a expansão do conceito de Medicina Baseada em Evidências determinou a hierarquização das recomendações clínicas, segundo o rigor metodológico dos estudos que as embasaram. Essa abordagem explícita vem ampliando a compreensão e a aplicação das recomendações clínicas. Por esses motivos, a AMIB - Associação de Medicina Intensiva Brasileira - e a SBPT - Sociedade Brasileira de Pneumologia e Tisiologia - julgaram conveniente a atualização das recomendações descritas no Consenso anterior. Dentre os tópicos selecionados a Fisioterapia durante a Ventilação Mecânica foi um

1. Professor do Curso de Fisioterapia da Universidade de Santo Amaro UTI Geral do Hospital Nossa Senhora de Lourdes (SP)

2. Laboratório de Pneumologia Experimental do Hospital das Clínicas da FMUSP. UTI - Geral do Hospital Nossa Senhora de Lourdes (SP)

3. Coordenador do Curso de Especialização em Fisioterapia Pneumofuncional da UCB/Atualiza (BA)

4. Professora do Curso de Fisioterapia da Universidade de São Paulo - Ribeirão Preto (SP)

5. UTI Geral do Hospital Sírio Libanês (SP)

6. Centro Universitário São Camilo (SP)

7. Professor do Curso de Fisioterapia na Universidade Federal do Rio de Janeiro (RJ)

8. Prof. do Curso de Fisioterapia da Universidade Metodista de São Bernado - SP

9. Fisioterapeuta da UTI do trauma do HCFMUSP (Hospital das Clínicas da Faculdade de Medicina da Universidade de São Paulo)

10. Prof. do Curso de Fisioterapia da Universidade Metodista de São Bernado (SP)

11. Centro Universitário São Camilo (SP)

12. UTI do Hospital AC Camargo e UTI-Respiratória do Hospital das Clínicas da FMAUSP (SP)

13. UTI do Hospital AC Camargo (SP)

(C)Associação de Medicina Intensiva Brasileira, 2007 dos temas propostos. O objetivo foi descrever os pontos mais importantes relacionados à atuação do fisioterapeuta no ambiente da terapia Intensiva com ênfase na ventilação mecânica.

MÉTODO: Objetivou-se chegar a um documento suficientemente sintético, que refletisse a melhor evidência disponível na literatura. A revisão bibliográfica baseouse na busca de estudos através de palavras-chave e em sua gradação conforme níveis de evidência. As palavras-chave utilizadas para a busca foram: mechanical ventilation e physical therapy.

RESULTADOS: São apresentadas recomendações quanto aos principais procedimentos fisioterápicos, as técnicas e suas aplicações.

CONCLUSÕES: A fisioterapia ocupa hoje papel relevante no ambiente da terapia intensiva, principalmente para os pacientes sob ventilação mecânica invasiva ou não invasiva.

Unitermos: fisioterapia respiratória, Ventilação mecânica não-invasiva, ventilação mecânica.

\section{SUMMARY}

BACKGROUND AND OBJECTIVES: The II Brazilian Consensus Conference on Mechanical Ventilation was published in 2000. Knowledge on the field of mechanical ventilation evolved rapidly since then, with the publication of numerous clinical studies with potential impact on the ventilatory management of critically ill patients. Moreover, the evolving concept of evidence - based medicine determined the grading of clinical recommendations according to the methodological value of the studies on which they are based. This explicit approach has broadened the understanding and adoption of clinical recommendations. For these reasons, AMIB - Associação de Medicina Intensiva Brasileira and SBPT - Sociedade Brasileira de Pneumologia e Tisiologia - decided to update the recommendations of the II Brazilian Consensus. Physical therapy during mechanical ventilation has been one of the updated topics. This 
objective was described the most important topics on the physical therapy during mechanical ventilation.

METHODS: Systematic review of the published literature and gradation of the studies in levels of evidence, using the key words: mechanical ventilation and physical therapy.

RESULTS: Recommendations on the most important techniques applied during mechanical ventilation.

CONCLUSIONS: Physical therapy has a central role at the Intensive Care environment, mainly in patients submitted to a mechanical ventilatory support invasive or non invasive.

Key Words: mechanical ventilation, physical therapy, noninvasive ventilation.

\section{INTRODUÇÃO}

A Fisioterapia faz parte do atendimento multidisciplinar oferecido aos pacientes em unidade de terapia intensiva (UTI). Sua atuação é extensa e se faz presente em vários segmentos do tratamento intensivo, tais como o atendimento a pacientes críticos que não necessitam de suporte ventilatório; assistência durante a recuperação pós-cirúrgica, com o objetivo de evitar complicações respiratórias e motoras; assistência a pacientes graves que necessitam de suporte ventilatório. Nesta fase, o fisioterapeuta tem importante participação, auxiliando na condução da ventilação mecânica, desde o preparo e ajuste do ventilador artificial à intubação, evolução do paciente durante a ventilação mecânica, interrupção e desmame do suporte ventilatório e extubação.

Neste Consenso, abordou-se exclusivamente a atuação do fisioterapeuta no tratamento dos pacientes sob ventilação mecânica invasiva e não-invasiva, baseando-se as recomendações em resultados de estudos clínicos e na opinião dos especialistas que expõem sua experiência na área de terapia intensiva.

Graus de recomendação (Quadro 1).
FISIOTERAPIA RESPIRATÓRIA DURANTE A VENTILAÇÃO MECÂNICA

\author{
Fisioterapia na Prevenção de Pneumonia Associa- \\ da à Ventilação Mecânica (PAV) \\ Fisioterapia Respiratória \\ Recomendação: A fisioterapia respiratória é recomen- \\ dada para a prevenção de pneumonia associada à \\ ventilação mecânica.
}

\section{Grau de Recomendação: C}

Comentário: Ntoumenopoulos e col. compararam em pequeno estudo controlado, mas não aleatório, fisioterapia respiratória (vibrocompressão e aspiração endotraqueal) com um grupo controle (sem fisioterapia respiratória). Observaram que apenas $8 \%$ dos pacientes do grupo intervenção desenvolveram PAV, comparado com $39 \%$ no grupo controle ${ }^{1}$.

\section{Posicionamento do Paciente}

Recomendação: $\mathrm{Na}$ ausência de contra-indicações, manter o decúbito elevado (entre $30^{\circ}$ e $45^{\circ}$ ) em pacientes em ventilação mecânica para prevenção de PAV, mesmo durante a fisioterapia motora.

Grau de Recomendação: B

Comentário: $\mathrm{O}$ decúbito elevado $\left(45^{\circ}\right)$ reduziu o risco de pneumonia associada à ventilação em relação à posição supina em estudo aleatório e controlado. Drakulovic e col. mostraram que a ocorrência de PAV foi menor no grupo do decúbito elevado quando comparado ao grupo em supino (decúbito elevado 2/39 [5\%] versus supino 11/47 [23\%]; OR 4,2 - 31,8, $\mathrm{p}=0,018)^{2}$.

Fisioterapia Respiratória no Tratamento da Atelectasia Pulmonar

Recomendação: A fisioterapia respiratória é eficaz e recomendada para o tratamento das atelectasias pulmonares em pacientes em ventilação mecânica.

Quadro 1 - Graus de Recomendação

\begin{tabular}{ll}
\hline Graus de Recomendação & Tratamento/Prevenção \\
\hline A & Revisão sistemática (com homogeneidade) de ensaios clínicos controlados e aleatórios; \\
& Ensaio clínico controlado e aleatório com Intervalo de confiança estreito; \\
& Resultados terapêuticos do tipo "tudo ou nada". \\
B & Revisão sistemática (com homogeneidade) de estudos de coorte; \\
& Estudo de coorte (incluindo ensaio clínico aleatório de menor qualidade); \\
& Observação de resultados terapêuticos (outcomes research) / estudo ecológico; \\
& Revisão sistemática (com homogeneidade) de estudos caso-controle; \\
& Estudo caso-controle. \\
C & Relato de casos (incluindo coorte ou caso-controle de menor qualidade). \\
D & Opinião de especialista sem avaliação crítica ou baseada em matérias básicas (estudo fisiológico ou es- \\
& tudo com animais). \\
\hline
\end{tabular}




\section{Grau de Recomendação: B}

Comentário: Ensaio clínico com 31 pacientes com diagnóstico radiológico de atelectasia, submetidos à broncoscopia e fisioterapia versus fisioterapia respiratória isoladamente, não mostrou diferença entre os grupos, demonstrando eficácia semelhante na resolução das atelectasias em pacientes ventilados de forma invasiva ${ }^{3}$.

\section{PROCEDIMENTOS FISIOTERÁPICOS}

A fisioterapia respiratória pode ser utilizada em pacientes críticos, com o objetivo de prevenir e/ou tratar complicações respiratórias. Para isso, geralmente é usada uma combinação dos procedimentos descritos a seguir, que objetivam a "re-expansão pulmonar" e a "remoção de secreções nas vias aéreas". O quadro 2 descreve os procedimentos de fisioterapia respiratória descritos na literatura para a terapêutica de pacientes em ventilação mecânica.

\section{Aspiração Traqueal \\ Freqüência}

Recomendação: A aspiração somente deverá ser realizada quando necessária, isto é, quando houver sinais sugestivos da presença de secreção nas vias aéreas (por exemplo, secreção visível no tubo, som sugestivo na ausculta pulmonar, padrão denteado na curva fluxovolume observado na tela do ventilador, etc.).

\section{Grau de Recomendação: D}

Comentário: A avaliação da necessidade de aspiração pelo fisioterapeuta deve ser sistemática, em intervalos fixos e, também, na presença de desconforto respiratório. A aspiração traqueal é um procedimento invasivo, bastante irritante e desconfortável para os pacientes. Pode ainda promover complicações, entre as quais tosse, broncoespasmo, hipoxemia, disritmias e lesões na mucosa ${ }^{4}$. Apesar de serem claros na prática clínica, os benefícios da aspiração para remoção das secreções das vias aéreas, nunca foi estudado ou, principalmente, avaliados os efeitos colaterais associados a ela ${ }^{5}$. Lesões na mucosa e no sistema mucociliar geralmente estão associados à técnica do operador e à quantidade de pressão usada (que não deve exceder $150 \mathrm{mmHg}$ em adultos) $)^{6}$. Aspiração intermitente, em vez de contínua, pode ser menos traumática para a mucosa, mas existe pouca evidência sobre isso ${ }^{6}$.

\section{Prevenção de Hipoxemia}

Recomendação: A hiper-oxigenação $\left(\mathrm{FIO}_{2}=1\right)$ deve ser utilizada previamente ao procedimento de aspiração traqueal para diminuir a hipoxemia induzida pela aspiração traqueal.

\section{Grau de Recomendação: A}

Comentário: A hiper-oxigenação com $\mathrm{FiO}_{2}$ de $100 \%$ associada à hiperinsuflação com VT $50 \%$ maior que o basal durante três a seis ciclos respiratórios foram

Quadro 2 - Procedimentos de Fisioterapia Utilizados durante a Ventilação Mecânica

\begin{tabular}{ll}
\hline Manobras & Descrição \\
\hline Aspiração & $\begin{array}{l}\text { Retirada passiva das secreções, com técnica asséptica, por um cateter conectado a um sistema } \\
\text { de vácuo, introduzido na via aérea artificial. }\end{array}$ \\
Percussão e vibração & $\begin{array}{l}\text { Procedimento manual aplicado sobre o tórax, que busca transmitir uma onda de energia através } \\
\text { da parede torácica e favorecer o deslocamento de secreçães. }\end{array}$ \\
Drenagem postural & $\begin{array}{l}\text { Posicionamento do corpo do paciente, de modo que o segmento pulmonar a ser drenado seja } \\
\text { favorecido pela ação da gravidade. }\end{array}$ \\
Compressão brusca do tórax & $\begin{array}{l}\text { Compressão vigorosa do tórax, no início da expiração espontânea ou da fase expiratória da ven- } \\
\text { tilação mecânica, a fim de obter um aumento do fluxo expiratório. }\end{array}$ \\
Posicionamento corporal & $\begin{array}{l}\text { Adequação da posição do corpo no leito como um tratamento específico, com o objetivo de oti- } \\
\text { mizar a relação ventilação/perfusão, aumentar o volume pulmonar, reduzir o trabalho ventilatório } \\
\text { e cardíaco e de aumentar a depuração mucociliar. }\end{array}$ \\
Expansão/re-expansão pulmonar & $\begin{array}{l}\text { Uso de procedimentos que aumentem a pressão e/ou volume alveolar, promovendo expansão de } \\
\text { unidades alveolares colabadas. }\end{array}$ \\
Desconexão do paciente do ventilador, seguida de insuflação pulmonar com um ressuscitador \\
manual, aplicando-se volume de ar maior do que o volume corrente utilizado. Freqüentemente, re- \\
alizam-se inspiraçães lentas e profundas, seguidas de pausa inspiratória e uma rápida liberação, \\
a fim de obter um aumento do fluxo expiratório. \\
Uso da técnica de pressão positiva ao final da expiração ou pressão positiva contínua nas vias \\
aéreas para promover expansão de unidades alveolares colabadas
\end{tabular}

PEEP = pressão positiva ao final da expiração 
as técnicas mais estudadas para prevenir a hipoxemia durante a aspiração?.

\section{Sistema de Aspiração Aberto Versus Fechado \\ Recomendação: Os sistemas de aspiração aberto e fechado são igualmente eficazes na remoção de se- creções. No entanto, o sistema fechado determina me- nor risco de hipoxemia, disritmias e de contaminação e deve ser preferido, principalmente em situações nas quais são usados valores de PEEP elevados, como na lesão pulmonar aguda.}

\section{Grau de Recomendação: B}

Comentário: A principal vantagem do sistema fechado é realizar a aspiração sem a desconexão do circuito do ventilador. Isso, além de determinar menor alteração hemodinâmica e nas trocas gasosas, poderia implicar em menor risco de infecção. Porém, os estudos realizados não mostraram menor freqüência de pneumonia com o sistema fechado ${ }^{8}$. No entanto, em pacientes com lesão pulmonar aguda / síndrome do desconforto respiratório agudo, o uso do sistema fechado pode reduzir o desrecrutamento e a diminuição na oxigenação do paciente ${ }^{9}$. Esse efeito pode ser influenciado pelo modo ventilatório em uso e pelos ajustes do ventilador ${ }^{10}$. Uma manobra de recrutamento após a aspiração pode diminuir os efeitos da aspiração traqueal ${ }^{9}$. O custo relacionado ao uso do sistema fechado pode ser reduzido com a troca a cada sete dias, ao invés de diariamente, sem aumentar o risco de infecção respiratória ${ }^{11}$.

\section{Hiperinsuflação Manual (HM)}

Recomendação: A HM está indicada em pacientes que apresentem acúmulo de secreção traqueobrônquica.

\section{Grau de Recomendação: B}

Comentário: A hiperinsuflação manual potencializa as forças de recolhimento elástico pulmonar, promovendo um aumento do pico de fluxo expiratório e, conseqüentemente, favorecendo o deslocamento de secreção acumulada nas vias aéreas. Maa e col. classificaram 23 pacientes em desmame difícil para receber $\mathrm{HM}$ ou fisioterapia respiratória padrão. Nesse estudo, a HM foi aplicada de oito a 13 ciclos por minuto, com pressão limitada em $20 \mathrm{cmH}_{2} \mathrm{O}$ por 20 minutos, com freqüência de três vezes por dia, durante 5 dias. Eles observaram discreta melhora em desfechos intermediários, como $\mathrm{PaO}_{2} / \mathrm{FIO}_{2}$ e complacência estática, porém sem efeito sobre desfechos clínicos ${ }^{12}$. Choi e Jones compararam a HM seguida de aspiração versus aspiração isoladamente em 15 pacientes com pneumonia associada à ventilação mecânica. Obtiveram melhora da complacência e redução da resistência, que persistiu por pelo menos 30 min após o procedimento ${ }^{13}$. Contudo, em outro estudo, a aplicação de hiperinsuflação manual associada ao decúbito lateral, com posterior aspiração traqueal, em pacientes com lesão pulmonar, não promoveu diferença significativa nos valores de complacência e oxigenação após 60 min ${ }^{14}$. Estudo realizado por Denehy apontou as controvérsias sobre a segurança e a eficácia da HM e sugeriu que, quando aplicada, deve-se limitar o pico de pressão a $40 \mathrm{cmH}_{2} \mathrm{O}$, por risco de barotrauma ${ }^{15}$.

\section{Compressão Brusca do Tórax}

Recomendação: A compressão brusca do tórax deve ser realizada em pacientes com ausência ou diminuição do reflexo de tosse e em pacientes com dificuldade de mobilizar secreção, especialmente aqueles com disfunção neuromuscular.

\section{Grau de Recomendação: C}

Comentário: Até o momento, os dados da literatura não permitem conclusões sobre o uso rotineiro da compressão torácica para otimizar a remoção de secreções em pacientes sob ventilação mecânica. A compressão brusca é descrita com freqüência no tratamento de pacientes com lesão medular ou que apresentem algum tipo de fraqueza muscular ${ }^{16}$. Em estudo controlado, no qual se comparou a aspiração traqueal com e sem a associação da compressão brusca do tórax (por $5 \mathrm{~min}$ ), evidenciou-se que, no grupo da compressão brusca do tórax, a quantidade de secreção aspirada foi maior do que no grupo que recebeu apenas aspiração traqueal, porém sem atingir valor estatisticamente significativo ${ }^{17}$.

\section{Drenagem Postural, Vibração e Percussão Torácica} Recomendação: A drenagem postural, a vibração e a percussão torácica devem anteceder a aspiração traqueal.

\section{Grau de Recomendação: D}

Comentário: Embora a efetividade da percussão em promover o transporte de secreções brônquicas tenha sido relatada em pacientes DPOC estáveis e em ventilação espontânea, não há descrição na literatura destes mesmos resultados em pacientes sob ventilação artificial. Os estudos clínicos que avaliaram os efeitos fisiológicos dessas manobras, quando aplicadas isoladamente, apresentaram dados inconclusivos, com métodos distintos aplicados a populações variáveis ${ }^{18}$. 
O Fisioterapeuta na Aplicação da Ventilação Mecânica Não-Invasiva (VMNI)

Recomendação: $O$ fisioterapeuta deve instituir e acompanhar a VMNI no ambiente da terapia intensiva.

\section{Grau de Recomendação: B}

Comentário: A aplicação da VMNI exige maior atenção da equipe da UTI, principalmente nas horas inicias da sua instituição ${ }^{19-21}$. Kramer e col. mostraram que durante as 8 horas iniciais da VMNI, o fisioterapeuta despendeu cerca de 60 min a mais na assistência dos pacientes em VMNI quando comparado com o grupo sob tratamento convencional22.

\section{O Fisioterapeuta na Assistência do Desmame da Ventilação Mecânica}

Recomendação: A triagem sistemática de pacientes aptos para a realização do teste de respiração espontânea deve ser realizada diariamente pelo fisioterapeuta da UTI, seguindo protocolo multidisciplinar da respectiva unidade. O fisioterapeuta deve realizar o teste de respiração espontânea nos pacientes aptos, identificando assim os elegíveis para a interrupção da ventilação mecânica.

\section{Grau de Recomendação: A}

Comentário: O fisioterapeuta tem importante papel na condução de protocolos de triagem de pacientes para interrupção da ventilação mecânica ${ }^{23}$. Ely e col. demonstraram que a avaliação diária da capacidade respiratória dos pacientes em VM pelo fisioterapeuta (grupo intervenção) diminuiu o tempo de VM em 1,5 dia e reduziu a morbidade dos pacientes. A média de duração da VM no grupo intervenção foi de 4,5 dias e no grupo controle foi de 6 dias $(p=0,003)^{24}$. Kollef e col. em estudo aleatório e controlado, mostraram que o desmame protocolado e guiado por fisioterapeutas (grupo intervenção) reduziu a duração da VM e aumentou a taxa de sucesso no desmame. O índice de sucesso no desmame foi significativamente maior para os pacientes do grupo intervenção (odds ratio 1,31; 95\% IC 1,15 a 1,50; $p=0,039$ ). Porém, a mortalidade hospitalar foi similar entre os dois grupos $(22,3 \% \text { versus } 23,6 \%)^{25}$.

As recomendações relacionadas ao teste de respiração espontânea estão detalhadas no capítulo de Desmame.

\section{Treino Específico dos Músculos Respiratórios}

\section{Uso da Sensibilidade do Ventilador como Forma de Treinamento}

Recomendação: O treinamento dos músculos respiratórios por meio da redução da sensibilidade de disparo dos ventiladores não é fisiológico e parece não representar vantagem na liberação do paciente do ventilador, não sendo recomendada por este Consenso.

\section{Grau de Recomendação: B}

Comentário: Somente um estudo utilizou a redução da sensibilidade de disparo para treino dos músculos inspiratórios em pacientes sob VM, desde o início da ventilação, com objetivo de abreviar o desmame da ventilação e reduzir a taxa de re-intubação. Foram avaliados 25 pacientes, 12 treinados duas vezes ao dia através do ajuste da sensibilidade do ventilador e 13 controles. Não houve redução no tempo de desmame da VM, assim como, no índice de re-intubações ${ }^{26}$.

Treino de Força dos Músculos Respiratórios por Meio do Uso de Dispositivos de Incremento de Carga para Facilitar o Desmame

Recomendação: Não há evidências de que o treinamento muscular, através do uso de dispositivos que proporcionam aumento de carga (threshold), facilite o desmame de pacientes em ventilação mecânica. Portanto, essa técnica não é recomendada para pacientes com dificuldade para o desmame.

\section{Grau de Recomendação: D}

Comentário: Não há estudos prospectivos, controlados e aleatórios que mostrem utilidade de dispositivos de aumento de carga para a facilitação do desmame dos pacientes da ventilação mecânica, sendo a evidência restrita a pequenas séries de $\operatorname{casos}^{27,28}$.

\section{Treino de Endurance dos Músculos Respiratórios}

Recomendação: $O$ treinamento de endurance dos músculos respiratórios pode ser considerado para pacientes em ventilação mecânica prolongada. A sua realização deve ser de forma progressiva e protocolada.

\section{Grau de Recomendação: D}

Comentário: O treinamento de endurance consiste no aumento progressivo de carga aos músculos respiratórios. Ao respirar espontaneamente (em tubo-T ou com baixos valores de pressão de suporte), o paciente está trabalhando, ao longo do tempo, contra uma carga imposta pela retirada do suporte ventilatório. No entanto, essa carga imposta aos músculos respiratórios pode estar gerando um trabalho muscular acima do limiar de fadiga e/ou levando ao seu desenvolvimento se não for instituído um método para aliviar a carga ${ }^{29}$. Vários estudos demonstraram que quando o paciente começa a apresentar sinais clínicos de intolerância à respiração espontânea, como o uso de músculos acessórios, ele está entrando na zona de fadiga ${ }^{29-31}$. 
O aumento progressivo do tempo de respiração espontânea, alternado com o suporte ventilatório suficiente para diminuir o trabalho respiratório do paciente abaixo do limiar de fadiga, promove aumento da endurance dos músculos respiratórios, permitindo assim o ganho de maior tempo de treinamento, o que por sua vez proporciona maior tempo de respiração espontânea ao paciente.

Assim, para a realização de um treinamento gradual criterioso, seguindo as considerações descritas, é importante a elaboração de um protocolo (Anexo 1) para estabelecer as condutas na tentativa de diminuir o tempo e interromper a ventilação mecânica nesse grupo de pacientes.

\section{CUIDADOS COM AS VIAS AÉREAS ARTIFICIAIS}

\section{Fixação do Tubo Traqueal}

Recomendação: A adequada fixação do tubo traqueal e a avaliação da sua posição são aspectos muito importantes no cuidado da via aérea e devem ser realizados sistematicamente pela equipe assistente.

\section{Grau de Recomendação: B}

Comentário: O método ideal de fixação do tubo deve permitir a sua menor movimentação possível, ser confortável para o paciente, permitir higiene oral, preservar a pele íntegra e ser de fácil aplicação ${ }^{32}$. A fixação deve ser realizada por duas pessoas, sendo uma responsável por segurar o tubo na posição correta, enquanto a outra realiza a fixação. O método tradicional para fixar o tubo traqueal é com o uso de fita adesiva ${ }^{33}$. Um dos problemas que se pode encontrar com a utilização da fita é a dificuldade em realizar higiene oral. Cadarços também podem ser usados, mas escaras podem surgir nos lobos das orelhas, sendo necessário então protegê-las ou evitar o seu contato com o cadarço. As lesões causadas por pressão do tubo nos lábios podem ser evitadas através do seu reposicionamento periódico. Deve-se estar sempre atento à cavidade oral, aos lábios e à pele ao redor da boca.

\section{Cuidados com o Balonete da Via Aérea Artificial}

Recomendação: A pressão do balonete do tubo traqueal deve ser monitorada diariamente e deve ser mantida entre 20 e $34 \mathrm{cmH}_{2} \mathrm{O}$ (15 e $25 \mathrm{mmHg}$ ).

Grau de Recomendação: D

Comentário: A função do balonete do tubo traqueal é selar a via aérea. Durante a ventilação mecânica, a sua pressão deve ser baixa o suficiente para permitir a perfusão da mucosa e alta o suficiente para prevenir o vazamento de ar e impedir a aspiração das secreções. Monitorar a pressão do balonete três vezes por dia parece contribuir para prevenir lesões isquêmicas e estenose traqueal ${ }^{34}$. Pressão contínua na parede traqueal acima da pressão de perfusão capilar (25 a $35 \mathrm{mmHg}$ ) pode comprometer o fluxo sangüíneo na mucosa. Como a pressão transmitida pelo balonete para a parede traqueal, usualmente, é menor do que a pressão no interior do balonete, $25 \mathrm{mmHg}\left(34 \mathrm{cmH}_{2} \mathrm{O}\right)$ é a máxima pressão aceitáve| ${ }^{35,36}$.

\section{UTILIZAÇÃO DE UMIDIFICADORES DURANTE A VENTILAÇÃO MECÂNICA}

\section{Condicionamento do Ar Inspirado na Ventilação Mecânica}

Recomendação: Nos pacientes em ventilação mecânica invasiva, a umidificação e o aquecimento adequados dos gases são imprescindíveis para assegurar a integridade das vias aéreas e adequada função mucociliar.

\section{Grau de Recomendação: D}

Comentário: Durante o suporte ventilatório invasivo, os mecanismos naturais de aquecimento e umidificação do ar inspirado são suprimidos. Nesse contexto, a umidificação e o aquecimento do ar podem ser realizados tanto ativamente, através de umidificadores aquecidos (UA), como passivamente, por meio de trocadores de calor e umidade (HME - Heat and moisture exchangers ${ }^{37}$. Os HME são divididos em três categorias: os higroscópicos, os hidrofóbicos e os mistos (higroscópicos-hidrofóbicos) ${ }^{38}$. Os HME com propriedades higroscópicas têm melhor qualidade de umidificação, quando comparados aos HME que possuem somente componente hidrofóbico. Este tipo de HME esteve associado à oclusão do tubo traqueal em alguns estudos ${ }^{39}$. Por outro lado, os HME com componentes hidrofóbicos funcionam também como filtros de bactérias.

\section{Eficácia dos Dispositivos de Umidificação}

Recomendação: Para umidificação dos gases durante a ventilação mecânica invasiva, tanto os umidificadores aquecidos (UA) como os trocadores de calor e umidades (HME) determinam bons resultados clínicos.

\section{Grau de Recomendação: B}

Comentário: Os UA garantem ótimo aquecimento e umidificação ${ }^{40}$. Desvantagens: (1) maior custo $^{41}$; (2) condensação do vapor de água no circuito de ventilação e no reservatório, com potencial de contaminação 
bacteriana; e (3) necessidade de suprimento de energia e de água ${ }^{42}$. Seu uso incorreto pode causar aquecimento e umidificação excessivos ou insuficientes, podendo levar à hiper ou hipotermia, lesão térmica de via aérea ou fluidificação insuficiente da secreção. Existem sistemas de umidificação que usam circuito com fio aquecido (de maior custo), que promovem aquecimento mais preciso do ar e previnem a condensação de água no circuito, reduzindo o consumo de água e podendo, potencialmente, reduzir o risco de infecção, quando comparado com circuito usualmente utilizado (sem fio aquecido). Um grande estudo multicêntrico acompanhou 369 pacientes e seguiu critérios rigorosos para diagnóstico de PAV, não encontrando diferença significativa na incidência de PAV entre os pacientes que utilizaram umidificador com circuito com fio aquecido e HME misto ${ }^{43}$. São contra-indicações relativas para o uso de HME: (1) secreções espessas, abundantes ou sanguinolentas, pois pode haver oclusão do HME, resultando em excessiva resistência, hiperinsuflação pulmonar e necessidade de repetidas trocas do dispositivo; (2) fístula broncopleural volumosa ou vazamento de ar através do balonete do tubo traqueal; (3) temperatura corporal menor do que $32^{\circ} \mathrm{C}$, pois o $\mathrm{HME}$ funciona passivamente e retorna somente uma porção do calor e umidade exalados ${ }^{41,44}$; (4) grande volume minuto espontâneo (> $10 \mathrm{~L} / \mathrm{min}$ ) ou grande volume-corrente podem diminuir a eficiência de umidificação dos $\mathrm{HME}^{45}$; e (5) durante tratamento com aerossol. Nesta situação, - HME deve ser removido do circuito do paciente durante a nebulização, pois a retenção do vapor de água e dos fármacos aerossóis pelo HME pode aumentar a resistência do circuito ${ }^{41}$. Dentre as possíveis complicações descritas na literatura decorrentes do uso dos HME estão: (1) o aumento da resistência ${ }^{46}$; (2) o aumento do trabalho da respiração ${ }^{47}$; e (3) a hipoventilação, devido ao aumento do espaço morto ${ }^{48}$.

\section{FISIOTERAPIA MOTORA NO PACIENTE SOB VEN- TILAÇÃO MECÂNICA}

\section{Exercícios Passivos}

Recomendação: Apesar da ausência de dados que demonstrem a importância da utilização do exercício passivo para evitar deformações articulares e encurtamento muscular em pacientes sob ventilação mecânica, recomenda-se a sua aplicação nos pacientes em ventilação mecânica invasiva.

\section{Grau de Recomendação: D}

Comentário: O imobilismo causa diversas complica- ções, como úlceras de decúbito, perda de força muscular, tromboembolismo, osteoporose e pneumonia ${ }^{49}$. Os pacientes críticos, especialmente os idosos, têm maior risco de desenvolver as complicações da síndrome da imobilidade. A eficácia dos exercícios passivos em prevenir alterações músculo-esqueléticas foi pouco estudada ${ }^{50}$.

\section{Exercícios Ativos}

Recomendação: Recomenda-se a realização de exercícios ativos em pacientes sob ventilação mecânica capazes de executá-los, na ausência de contra-indicações, com o objetivo de diminuir a sensação de dispnéia, aumentar a tolerância ao exercício, reduzir a rigidez e dores musculares e preservar a amplitude articular.

\section{Grau de Recomendação: C}

Comentário: Há benefícios do uso de exercícios ativos de membros nos pacientes em desmame e recémliberados da ventilação mecânica. Uma abordagem multiprofissional que estimulou a mobilização precoce de pacientes em pós-operatório de cirurgias de aorta abdominal resultou em diminuição da morbidade e do tempo de internação'. Mais recentemente, um estudo prospectivo, controlado e aleatório analisou os efeitos do treino precoce em 66 pacientes desmamados de VM entre 48 e $96 \mathrm{~h}$. A intervenção consistia em treinamento nos membros superiores e fisioterapia global comparada com fisioterapia global isolada. Concluíram que o treino nos membros superiores era praticável em pacientes recentemente desmamados e que pode realçar os efeitos da fisioterapia global, sendo a função dos músculos inspiratórios relacionados com a melhora da capacidade de exercícios ${ }^{51}$. Estudo fisiológico prospectivo e controlado teve o objetivo de avaliar os efeitos do treino nos membros superiores com e sem o suporte ventilatório em pacientes portadores de DPOC, com dificuldade para o desmame. Encontraram um aumento da tolerância do exercício quando os pacientes o realizaram durante o suporte ventilatório ${ }^{52}$.

\section{Ortostatismo}

Recomendação: A posição ortostática como recurso terapêutico pode ser adotada de forma ativa ou passiva para estimulação motora, melhora da troca gasosa e do estado de alerta. Deve ser utilizada apenas em pacientes crônicos, estáveis clinicamente sob ventilação mecânica prolongada.

\section{Grau de Recomendação: D}

Comentário: A adoção da postura ortostática com assistência da prancha é recomendada para readaptar os 
pacientes à posição vertical, quando esses são incapazes de se levantar ou mobilizar com segurança, mesmo com considerável assistência ${ }^{53}$. O uso da postura ortostática na UTI tem sido encorajado como técnica para diminuir os efeitos adversos da imobilização prolongada ${ }^{54}$. Apesar da falta de ensaios clínicos avaliando o impacto no prognóstico nos pacientes críticos, a posição ortostática foi incluída como modalidade de tratamento em recente consenso por fisioterapeutas inglese ${ }^{55}$. Seus supostos benefícios incluem melhora no controle autonômico do sistema cardiovascular, facilitação da ventilação e troca gasosa, facilitação do estado de alerta, estimulação vestibular e facilitação da resposta postural antigravitacional ${ }^{56-59}$.

Anexo 1 - Sugestão de Protocolo de Treinamento de Endurance dos Músculos Respiratórios

Tolerância respiração espontânea

$<$ que 15 minutos

$>$ que 15 minutos

Período de Treino Alternando Carga (C) e Repouso (R)

Manhã

Dia 1 - 15 min de $\mathrm{C}$ com 60 min de $\mathrm{R}$

Dia 2 - 30 min de $C$ com 60 min de $R$

Dia 3 - 60 min de C com 60 min de R

Dia 4 - 90 min de $C$ com 60 min de $R$

Dia 6- 120 min de $\mathrm{C}$ com 60 min de $\mathrm{R}$

Dia 7- 180 min de $\mathrm{C}$ com 60 min de $\mathrm{R}$

Dia 8- 180 min de $\mathrm{C}$ com 60 min de $\mathrm{R}$

Permitir respiração espontânea máxima, até iniciar $\mathrm{C}$ com músculos acessórios ou descon-

forto (zona de fadiga). Avaliar o tempo limite, por exemplo, mais 10 minutos.

Considerar a liberação do ventilador quando preencher os critérios de desmame

$\begin{array}{cc}\begin{array}{c}\text { Métodos de Treino } \\ \text { Carga } \\ \text { PSV de } 5 \mathrm{cmH}_{2} \mathrm{O} \\ \text { Tubo-T } \\ \text { Tarde }\end{array} & \begin{array}{c}\text { Repouso } \\ \text { Repouso PSV* } \\ \text { Repouso PSV* }\end{array} \\ \text { Repete manhã } & \text { Noite } \\ \text { Repete manhã } & \text { Repouso } \\ \text { Repete manhã } & \text { Repouso } \\ \text { Repete manhã } & \text { Repouso } \\ \text { Repete manhã } & \text { Repouso } \\ \text { Repete manhã } & \text { Repouso } \\ \text { Repete manhã } & \text { Repouso }\end{array}$

6 horas de $\mathrm{C}$ com 6 horas de $\mathrm{R}$

*Ajustar PSV para melhor sincronia e conforto

\section{REFERÊNCIAS}

01. Ntoumenopoulos G, Presneill JJ, McElholum M et al - Chest physiotherapy for the prevention of ventilator-associated pneumonia. Intensive Care Med, 2002;28:850-856.

02. Drakulovic MB, Torres A, Bauer TT et al - Supine body position as a risk factor for nosocomial pneumonia in mechanically ventilated patients: a randomised trial. Lancet, 1999;354:(9193):1851-1858.

03. Marini JJ, Pierson DJ, Hudson LD - Acute lobar atelectasis: a prospective comparison of fiberoptic bronchoscopy and respiratory therapy. Am Rev Respir Dis, 1979;119:971-978.

04. Guglielminotti J, Alzieu M, Maury E et al - Bedside detection of retained tracheobronchial secretions in patients receiving mechanical ventilation: is it time for tracheal suctioning? Chest, 2000;118:1095-1099.

05. Stiller K - Physiotherapy in intensive care: towards an evidence-based practice. Chest, 2000;118:1801-1813.

06. AARC clinical practice guideline. Endotracheal suctioning of mechanically ventilated adults and children with artificial airways. American Association for Respiratory Care. Respir Care, 1993;38:500-504.

07. Oh H, Seo W - A meta-analysis of the effects of various interventions in preventing endotracheal suction-induced hypoxemia. J Clin Nurs, 2003;12:912-924.

08. Lorente L, Lecuona M, Martin MM et al - Ventilator-associated pneumonia using a closed versus an open tracheal suction system. Crit Care Med, 2005;33:115-119.

09. Lasocki S, Lu Q, Sartorius A et al - Open and closed-circuit endotrachea suctioning in acute lung injury: efficiency and effects on gas exchange. Anesthesiology, 2006;104:39-47.

10. El Masry A, Williams PF, Chipman DW et al - The impact of closed endotracheal suctioning systems on mechanical ventilator performance. Respir Care, 2005;50:345-353.

11. Stoller JK, Orens DK, Fatica C et al - Weekly versus daily changes of in-line suction catheters: impact on rates of ventilator-associated pneu- monia and associated costs. Respir Care, 2003;48:494-499.

12. Maa SH, Hung TJ, Hsu $\mathrm{KH}$ et al - Manual hyperinflation improves alveolar recruitment in difficult-to-wean patients. Chest, 2005;128:27142721.

13. Choi JS, Jones AY - Effects of manual hyperinflation and suctioning in respiratory mechanics in mechanically ventilated patients with ventilatorassociated pneumonia. Aust J Physiother, 2005;51:25-30.

14. Ntoumenopoulos G, Gild A, Cooper DJ - The effect of manual lung hyperinflation and postural drainage on pulmonary complications in mechanically ventilated trauma patients. Anaesth Intensive Care, 1998;26:492496.

15. Denehy $L$ - The use of manual hyperinflation in airway clearance. Eur Respir J, 1999;14:958-965.

16. Slonimski M, Aguilera EJ - Atelectasis and mucus plugging in spinal cord injury: case report and therapeutic approaches. J Spinal Cord Med, 2001;24:284-288.

17. Unoki T, Kawasaki $\mathrm{Y}$, Mizutani $\mathrm{T}$ et al - Effects of expiratory rib-cage compression on oxygenation, ventilation, and airway-secretion removal in patients receiving mechanical ventilation. Respir Care, 2005;50:14301437.

18. Hess DR - The evidence for secretion clearance techniques. Respir Care, 2001;46:1276-1293.

19. Evans TW - International Consensus Conferences in Intensive Care Medicine: non-invasive positive pressure ventilation in acute respiratory failure. Organised jointly by the American Thoracic Society, the European Respiratory Society, the European Society of Intensive Care Medicine, and the Societe de Reanimation de Langue Francaise, and approved by the ATS Board of Directors, December 2000. Intensive Care Med, 2001;27:166-178.

20. Hill NS - Noninvasive ventilation for chronic obstructive pulmonary disease. Respir Care, 2004;49:72-89.

21. Hess DR - The evidence for noninvasive positive-pressure ventilation in the care of patients in acute respiratory failure: a systematic review of the literature. Respir Care, 2004;49:810-829. 
22. Kramer N, Meyer TJ, Meharg $\mathrm{J}$ et al - Randomized, prospective trial of noninvasive positive pressure ventilation in acute respiratory failure. Am J Respir Crit Care Med, 1995;151:1799-1806.

23. Ely EW, Meade MO, Haponik EF et al - Mechanical ventilator weaning protocols driven by nonphysician health-care professionals: evidence-based clinical practice guidelines. Chest, 2001;120:(Suppl6):454S463S.

24. Ely EW, Baker AM, Dunagan DP et al - Effect on the duration of mechanical ventilation of identifying patients capable of breathing spontaneously. N Engl J Med, 1996;335:1864-1869.

25. Kollef $\mathrm{MH}$, Shapiro SD, Silver $\mathrm{P}$ et al - A randomized, controlled trial of protocol-directed versus physician-directed weaning from mechanical ventilation. Crit Care Med, 1997;25:567-574.

26. Caruso P, Denari SD, Ruiz SA et al - Inspiratory muscle training is ineffective in mechanically ventilated critically ill patients. Clinics, 2005;60:479-484.

27. Martin AD, Davenport PD, Franceschi AC et al - Use of inspiratory muscle strength training to facilitate ventilator weaning: a series of 10 consecutive patients. Chest, 2002;122:192-196.

28. Sprague SS, Hopkins PD - Use of inspiratory strength training to wean six patients who were ventilator-dependent. Phys Ther, 2003;83:171-181.

29. Brochard L, Harf A, Lorino H et al - Inspiratory pressure support prevents diaphragmatic fatigue during weaning from mechanical ventilation. Am Rev Respir Dis, 1989;139:513-521.

30. Vassilakopoulos T, Zakynthinos S, Roussos C - The tension-time index and the frequency/tidal volume ratio are the major pathophysiologic determinants of weaning failure and success. Am J Respir Crit Care Med, 1998;158:378-385.

31. Laghi F, Cattapan SE, Jubran A et al - Is weaning failure caused by low-frequency fatigue of the diaphragm? Am J Respir Crit Care Med, 2003;167:120-127.

32. Hess DR - Managing the artificial airway. Respir Care, 1999;44:759772.

33. Patel N, Smith CE, Pinchak AC et al - Taping methods and tape types for securing oral endotracheal tubes. Can J Anaesth 1997;44:330-336.

34. Bernhard WN, Yost L, Joynes D et al - Intracuff pressures in endotracheal and tracheostomy tubes. Related cuff physical characteristics. Chest, 1985;87:720-725.

35. Mehta S, Mickiewicz M - Pressure in large volume, low pressure cuffs: its significance, measurement and regulation. Intensive Care Med, 1985;11:267-272.

36. Sengupta P, Sessler DI, Maglinger P et al - Endotracheal tube cuff pressure in three hospitals, and the volume required to produce an appropriate cuff pressure. BMC Anesthesiol, 2004;4:8.

37. AARC clinical practice guideline. Humidification during mechanical ventilation. American Association for Respiratory Care. Respir Care, 1992;37:887-890.

38. Ricard JD, Le Miere E, Markowicz P et al - Efficiency and safety of mechanical ventilation with a heat and moisture exchanger changed only once a week. Am J Respir Crit Care Med, 2000;161:104-109.

39. Villafane MC, Cinnella G, Lofaso F et al - Gradual reduction of endotracheal tube diameter during mechanical ventilation via different humidification devices. Anesthesiology, 1996;85:1341-1349.

40. Holt TO - Aerosol Generators and Humidifiers, em: Barnes TA - Core Textbook of Respiratory Care Practice. $2^{\text {nd }}$ Ed, St. Louis: Mosby, 1994;441-84.
41. Hess DR, Branson RD - Humidification, em: Branson RD, Hess DR, Chatburn RL - Respiratory Care Equipment. $2^{\text {nd }} \mathrm{Ed}$, Philadelphia: Lippincott Williams \& Wilkins; 1999;101-32.

42. Branson RD, Campbell RS, Johannigman JA et al - Comparison of conventional heated humidification with a new active hygroscopic heat and moisture exchanger in mechanically ventilated patients. Respir Care, 1999;44:912-917.

43. Lacherade JC, Auburtin M, Cerf C et al - Impact of humidification systems on ventilator-associated pneumonia: a randomized multicenter trial. Am J Respir Crit Care Med, 2005;172:1276-1282.

44. Lellouche F, Maggiore SM, Deye $\mathrm{N}$ et al - Effect of the humidification device on the work of breathing during noninvasive ventilation. Intensive Care Med, 2002;28:1582-1589.

45. Unal N, Kanhai JK, Buijk SL et al - A novel method of evaluation of three heat-moisture exchangers in six different ventilator settings. Intensive Care Med, 1998;24:138-146.

46. Lucato JJJ, Tucci MR, Schettino GPP et al - Evaluation of resistance in eight different heat and moisture exchangers: effects of saturation and flow rate/profile. Respir Care, 2005;50:636-643.

47. Girault C, Breton L, Richard JC et al - Mechanical effects of airway humidification devices in difficult to wean patients. Crit Care Med, 2003;31:1306-1311.

48. Jaber S, Chanques G, Matecki S et al - Comparison of the effects of heat and moisture exchangers and heated humidifiers on ventilation and gas exchange during non-invasive ventilation. Intensive Care Med, 2002;28:1590-1594.

49. Allen C, Glasziou P, Del Mar C - Bed rest: a potentially harmful treatment needing more careful evaluation. Lancet, 1999;354(9186):1229-1233.

50. Nava S, Piaggi G, De Mattia E et al - Muscle retraining in the ICU patients. Minerva Anestesiol, 2002;68:341-345.

51. Porta R, Vitacca M, Gile LS et al - Supported arm training in patients recently weaned from mechanical ventilation. Chest, 2005;128:25112520.

52. Vitacca M, Bianchi L, Sarva M et al - Physiological responses to arm exercise in difficult to wean patients with chronic obstructive pulmonary disease. Intensive Care Med, 2006;32:1159-1166.

53. Webber BPJ - Physiotherapy for Respiratory and Cardiac Problems, em: Webber BP - Physiotherapy Skills: Techniques and Adjuncts. Edinburgh: Churchill Livingstone; 1993.

54. Szaflarski N - Immobility Phenomena in Critically ill Adults, em: Clochesy JBC, Cardin SR - Critical Care Nursing. Philadelphia: Saunders; 1993;31-54.

55. Group AaHA - The Role of Healthcare Professionals Within Critical Care Services. Agency NM, 2002;16-17.

56. Chang AT, Boots RJ, Hodges PW et al - Standing with the assistance of a tilt table improves minute ventilation in chronic critically ill patients. Arch Phys Med Rehabil, 2004;85:1972-1976.

57. Egana M, Green S - Effect of body tilt on calf muscle performance and blood flow in humans. J Appl Physiol, 2005;98:2249-2258.

58. Tyson SF, Nightingale P - The effects of position on oxygen saturation in acute stroke: a systematic review. Clin Rehabil, 2004;18:863-871.

59. Winker R, Barth A, Bidmon D et al - Endurance exercise training in orthostatic intolerance: a randomized, controlled trial. Hypertension, 2005;45:391-398. 